\title{
DEPLOYING A FRAMEWORK TO IMPROVE THE PERFORMANCE OF A WEB SERVICE
}

\author{
S. Goyal \\ $\mathrm{PhD}$ Scholar, Department of Computer Science and Engineering, \\ JECRC University, Jaipur, India
}

A. Gill

Assistant Professor, Department of Electrical Engineering, JECRC University, Jaipur, India

\begin{abstract}
Information technology is being used to grow the business and every business has its own website. However, many websites lose customers to their slow speed during this fast-moving life. Sometimes this speed lag could be of milliseconds or seconds; this research paper aims to create a framework that can improve the availability and performance of such web services. The framework designed for this research strictly works on reducing the response time and cost of these services. Also, this framework help to build services or website High-availability, scalable, durable, and balance all traffic load which comes on web app or services. This happened with the help of Kubernetes. The best way of achieving this is often using the containerized approach with Kubernetes together with a further cache layer within the architecture for reducing response time. The framework is tested and compared to the already existing and successful frameworks. The results discussed during this paper provide insights that have contributed to achieving a much better response time with a minimum possible cost.
\end{abstract}

Key words: Containers, Kubernetes, Redis Cache, Response Time, LAMP

Cite this Article: S. Goyal and A. Gill, Deploying a Framework to Improve the Performance of a Web Service, International Journal of Electrical Engineering and Technology (IJEET), 12(5), 2021, pp. 137-147.

https://iaeme.com/Home/issue/IJEET?Volume $=12 \&$ Issue $=5$

\section{INTRODUCTION}

When you're online, the clock is always ticking. Every single second counts. Hence, the primary impression an internet site makes on its users is by its speed [1]. By the terms of human needs and not the technologies, the basic user interface design rule is the responsiveness. A website where a user can surf freely and not waste time endlessly waiting for the page to be loaded, would engage more audience than the websites with the poor response time. The poor 
response time of the website can result in a reduction in the number of users. For example, if some business website or an online retailer's website takes more time to respond, many customers might be unhappy, and it can range to negative brand perception as well resulting to a massive loss in the number of buyers. Google performed a research that remarked that 53\% of the users would abandon a website if it takes more than 3 seconds to load. Amazon also performed tests which showed that the company would suffer the losses of approximately $\$ 1.6$ Billion annually if their website's response time increases just by one second [2]. The response time plays a significant role in attracting the users, the lower, and the better. This research paper focuses on developing a framework to improve the performance of the websites, mainly by reducing the response time. There are many approaches kept in focus to achieve this objective and are discussed later in this paper. The literature review discusses the various studies that are conducted to develop the framework. Further, the methods and process of achieving the desired objective are described further the analysis of the obtained results were discussed and finally, concludes the outcomes of the research.

\section{LITERATURE SURVEY}

Containers are an executable software unit that runs the application reliably on moving from one computing environment to another. The containers consist of the entire runtime environment bundled into one package that includes the application, its dependencies, the required libraries, binary codes and all the configuration files that are needed to be run on it. Plus, they do not have an operating system image which makes these containers more portable and lightweight decreasing the overhead [3]. Containerization allows development teams to deploy the applications more rapidly and operate them at an unremarkable scale. Kubernetes Containerized applications can get complicated at times. In containerized architecture, numerous separate containers are deployed across a cluster of virtual or physical machines while in production. This brings about to the need for container orchestration and Kubernetes is a tool for the same, which deploys and manages the applications that involve multiple containers. In other words, $\mathrm{K} 8 \mathrm{~s}$ is an open source platform that provides automation for many manual processes that involve deployment, management and scaling of containerized applications. There are various benefits of using Kubernetes for orchestrating containers. If any of the applications have broken, it will be self-healed with the following features like autoscaling, auto-replicating, auto-replacing, and auto-restarting. K8s also manages hardware resource allocation in order to utilize the resources to the maximum, including storage, memory and network bandwidth. Load balancing is also served by Kubernetes. Redis Cache - Redis is an in-memory data structure store which is an open source. It can be used as a message broker, database and cache. Using Redis as a cache provides outstanding performance. The in-memory dataset in Redis helps it to achieve this performance. As the in-memory data storage stores the data in the main memory itself, the need to access the disks is eliminated, hence attaining the minimal response time. But this might also lead to a risk of data loss which can be resolved by data persistence [4]. The dataset can be preserved by dumping it to the disk on a timely basis or simply each command can be appended to a log. In case of a networked, feature-rich, inmemory cache, the persistence can be disabled optionally. When using the Redis cache, as new data is added, the older data is automatically evicted according to memory utilisation.

LAMP is a solution stack that is used to build web sites and web applications. It has four open-source components including Linux operating system, Apache HTTP Server, MySQL relational database management system and the interpreter for Php programming language. These components are interchangeable [5].

XAMPP was developed by Apache Friends, XAMPP is an open-source web server solution. XAMPP stands for Cross-platform, Apache, MariaDB (MySQL), PHP and Perl (it has the 
interpreters for scripts written in these programming languages), i.e., it mainly consists of the above stated components. This is one of the lightweight and simplest servers that can be used to test the websites on localhost itself. Moreover, there are no restrictions on number of testing to be performed [6].

WAMP is another server that is a solution stack created by Romain Bourdon for the Microsoft Windows operating system. Apart from Windows, it consists of Apache web server, MySQL database and the interpreter for Php programming language, hence the name WAMP. It also has OpenSSL for the SSL support. Due to the Apache server, the websites / web pages can be tested on this local server without having them published live [7].

\section{METHODOLOGY}

The Kube Devops framework (proposed framework) was designed to improve the performance of the websites. Reducing the response time of the websites is the best way for performance optimization. The lowering of the response time is the result of inbuilt load balancing and autoscaling. To serve this purpose, containerized approach was used with Kubernetes and an additional cache layer. The server was working in this cache storage itself. Caching makes the accessing of HTML documents faster, hence improving the response time [8]. To commence with the methodology, framing the design and structure was the first step. The basic framework designing steps involved were:

- Installation

- Kubernetes master deployment

- Slave deployment

- Cross checking if the Kube Devops framework (proposed) was deployed successfully.

Using these steps, the framework was set up. The following were the description of the setup steps.

Step I: Disabling the swap - Kubernetes requires the swap to be disabled because Kubernetes tightly pack the instances for $100 \%$ utilization (approximately). This was done for the performance purpose as swap slows down things. All the deployments in K8s must be pinned with the memory/CPU limits so that a pod should never use swap when the schedular sends it to a machine.

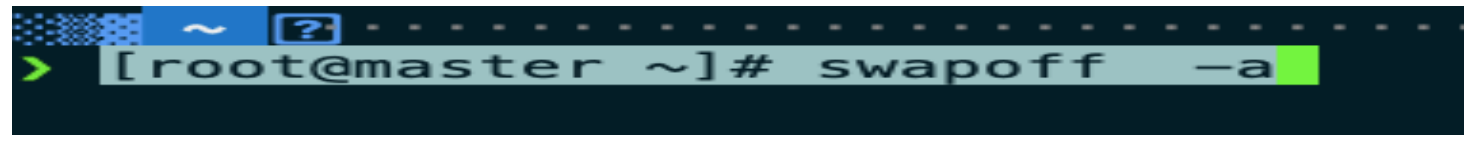

Figure 1. Step I of Framework Setup

Step II: Configuration of iptables so that the bridged network traffic could be received.

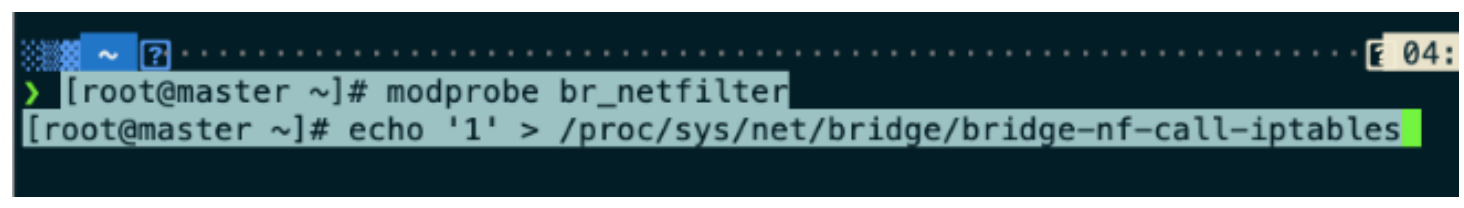

Figure 2. Step II of Framework Setup 
Step III: Creating the repository for Kubernetes installation to the system.

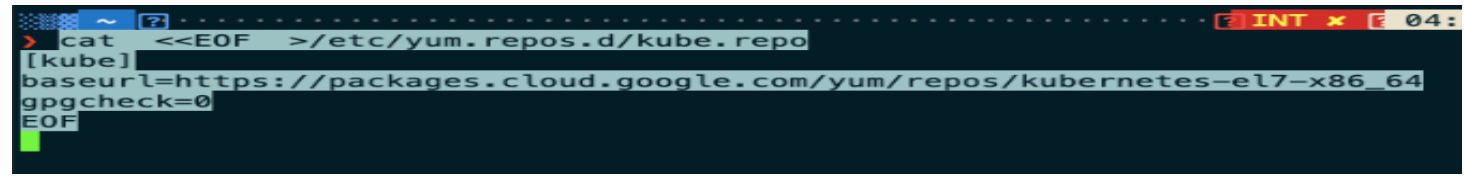

Figure 3. Step III of Framework Setup

Step IV: Installing docker and kubeadm.

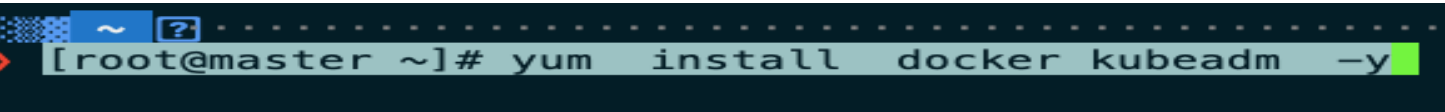

Figure 4. Step IV of Framework Setup

Step V: The below command was run in order to enable the docker and kubelet services automatically every time the system boots.

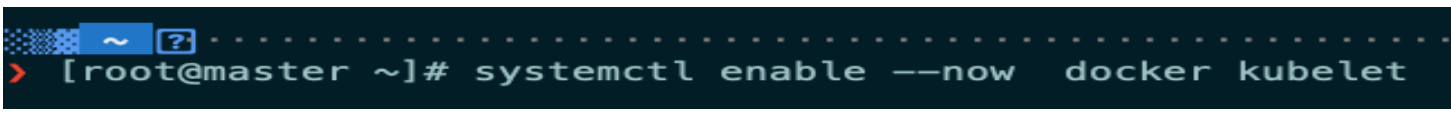

Figure 5. Step V of Framework Setup

Step VI: The cluster was created using kubeadm in it. The Calico network provider was used for this purpose and the pod-network-cidr switch was added to kubeadm in it as command line argument.

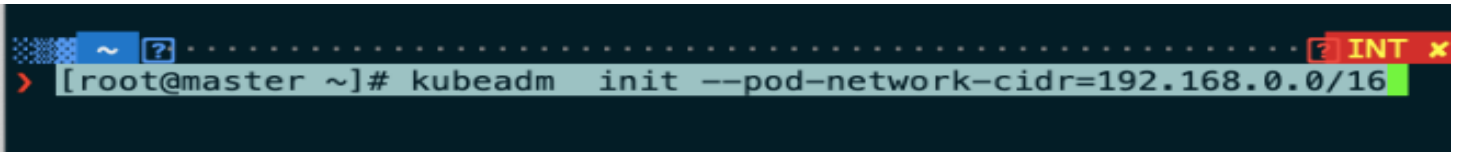

Figure 6. Step VI of Framework Setup

Step VII: Preparing the system for adding the network plugins and workloads.

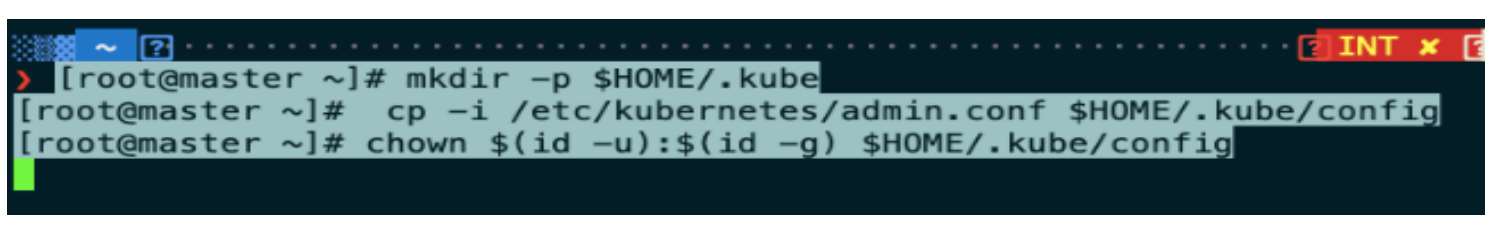

Figure 7. Step VII of Framework Setup

Step VIII: Installation of Calico plugin.

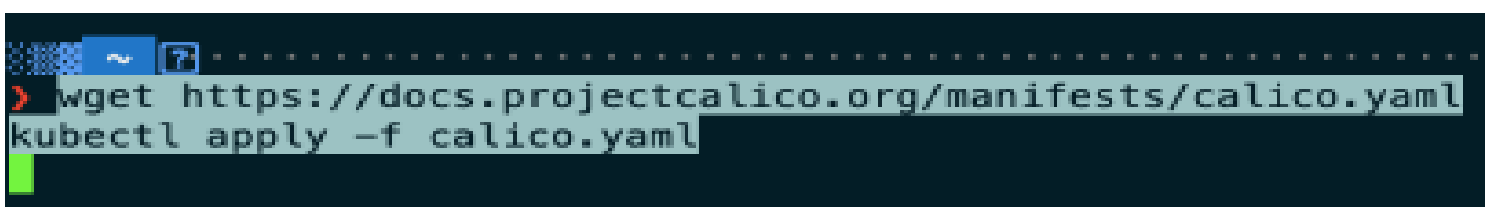

Figure 8. Step VIII of Framework Setup

Step IX: Testing of the Cluster. The figure below displays all four nodes which were the part of the cluster including the master node and the other worker nodes. And all the nodes were in Ready state, which states that a cluster's health is fine. 


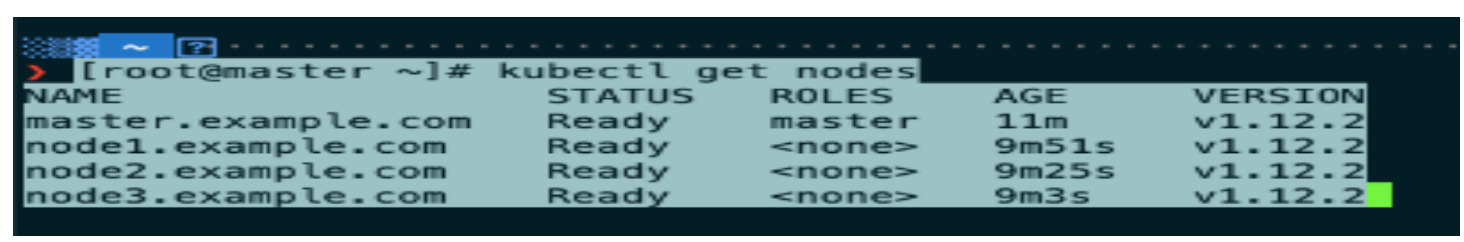

Figure 9. Step IX of Framework Setup

The architecture of the proposed framework shown in Fig 1 explains its internal structure and how the layers have been deployed. It depicts the master deployment and slave deployment of the Kubernetes layer.

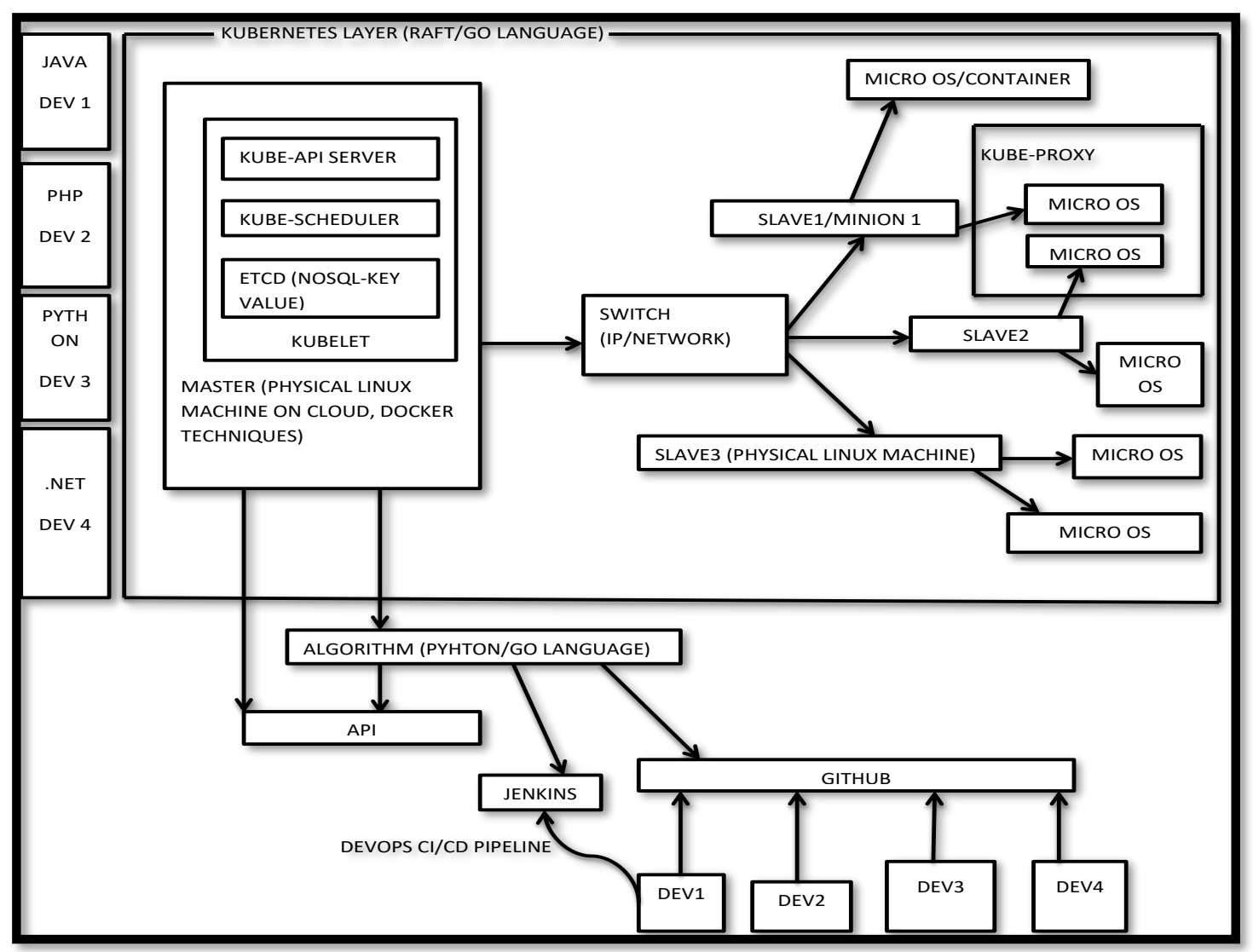

Figure 10. Kube Devops (Proposed) Framework Architecture

Containerization - To speed up the performance of the framework, containerized approach was used. The containers were light weighted as they do not contain any OS image. This lightweight property helps reduce the time taken by a web server to respond. Orchestrating the Containers - And to orchestrate these containers, the platform used was Kubernetes. The most important benefit of using Kubernetes for this framework was the autoscaling feature. A script was added to maintain the response time of the web services. For example, when the framework start, a container was launched along with it and it gives a responses time of 2 microseconds. As the number of users increase, the response time increases as well. As soon as the response time increases, another container was launched even though it's not needed immediately. Similarly, the containers were being scaled automatically in order to maintain the response time. This autoscaling feature also leads to load balancing as the sole need of autoscaling arose to balance the load of the web servers. Adding a cache layer - Traditionally, whenever a request was made for a service, the server searches and responds to it. A Redis cache was added so that for the repeated requests, the framework will not reload the server; instead it directly accesses 
the data from this cache. This reduces the response time of the web services. Generally, a web server accesses the hard disk to extract data but adding the cache layer completely saves this amount of time. The container directly reads from the cache which is faster than the traditional method. Improving the response time alone can become easier and simpler without additional constraints. For example, it can simply be improved just by enhancing the servers which, perhaps, can increase the cost [9] Hence, optimizing the cost along with becomes another challenge. Reducing the Cost - Normally, an instance was created on the cloud for the app deployment. When the load increases, a new instance was created (scaling up) for balancing the load. Hence, the cost is doubled. But for the proposed framework, the master-slave architecture is followed [10]. The application was deployed on a Kubernetes pod, hence for the purpose of load balancing the master node enables auto scaling and an exact copy of the existing pod was created.

\section{RESULTS AND DISCUSSION}

The above described approaches and techniques used for reducing the response time and improving the performance of the websites provided immense results. To deduce to the point that the proposed framework provides better response time, different websites were tested on various frameworks like cloud, localhost, WAMP, XAMPP and LAMPP, using the software Apache Benchmark. Input - Mainly, three websites were provided separately as input for the execution, HTML, PHP and Flask. A number of requests sent per test were approximately 1000 and the length of each document was of 612 bytes. The testing steps after the successful setup of the proposed framework (Kube Devops Framework) were shown in figures 11, 12 and 13.

Step 1: Checking for the details of the running pods and services using the kubectl utility.

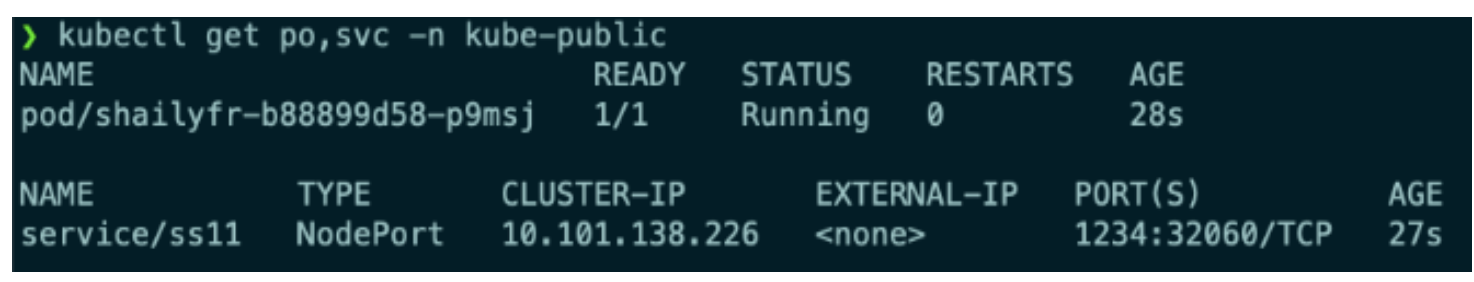

Figure 11. Checking for the Services and Pods

Step 2: Shelling into the bash terminal of the pod. This was done using the exec command of the kubectl utility. Post this, all the commands run directly inside the service deployed in that particular pod until exit.

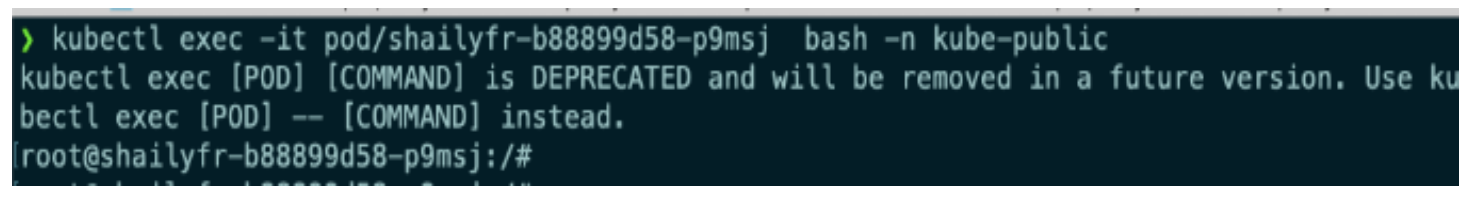

Figure 12. Shelling into the Pod Terminal

Step 3: This is the final command wherein 1000 requests were sent to the localhost server so that the execution time, response time and other factors could be observed and monitored in order to estimate the performance of the framework.

\section{root@shailyfr-b88899d58-p9msj:/usr/share/nginx/html\# ab -n 1000 -c 20 http://localhost/}

Figure 1. Sending Requests for executing at the Local host

After performing Step 3, the result was displayed on the command prompt. First all the requests were undertaken, and the actions were performed by the Apache Benchmark. After the 
completion of all the requests, the software outputs the status of the performance factors, like number of requests that were completed successfully as well as those which couldn't be completed and the time taken for the same, the details of the server and the document and many others which were described in the snapshot of the output below.

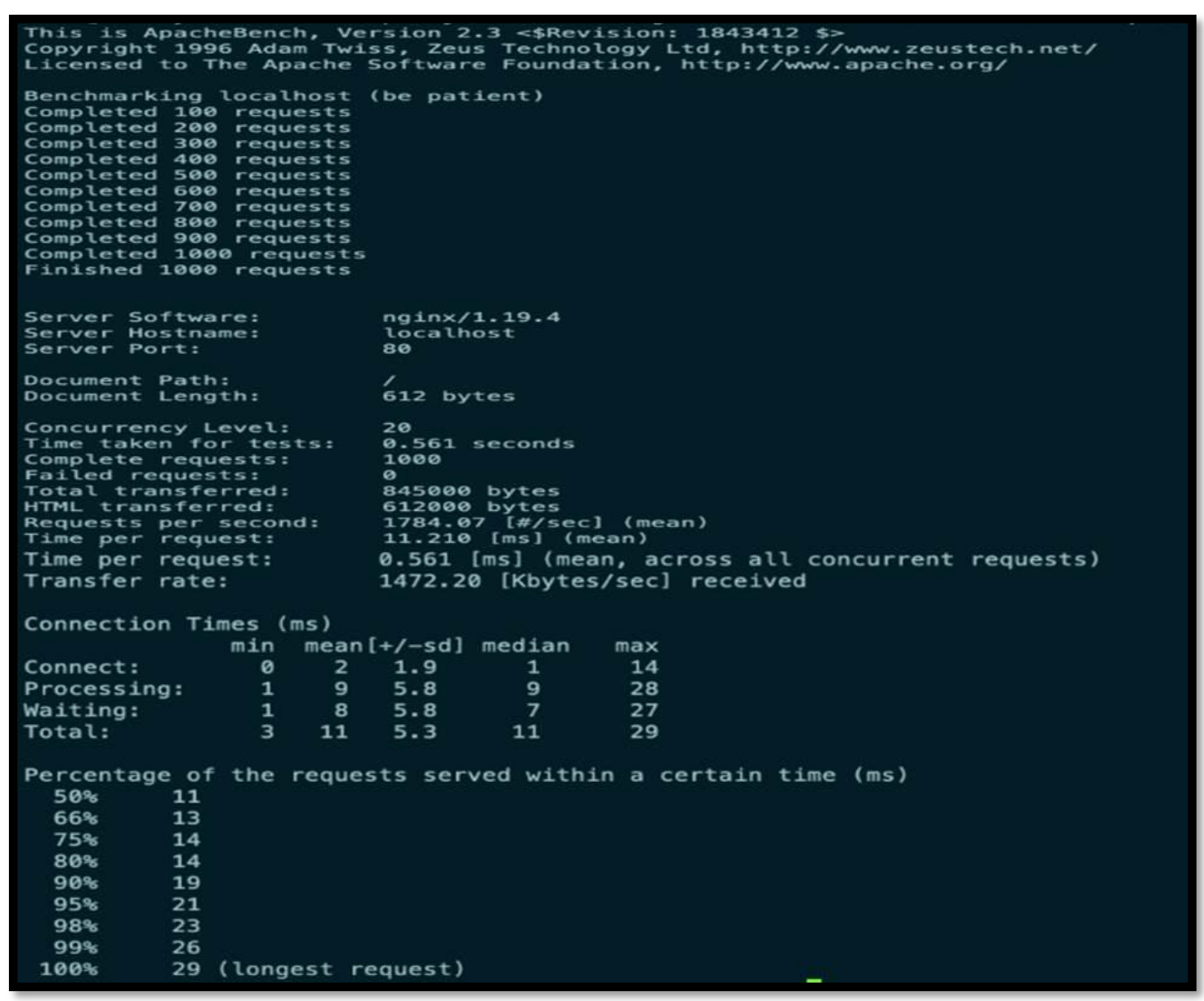

Figure 14. Performance Measure of the Proposed Framework

It can be perceived from the above figure that the proposed framework takes $11.210 \mathrm{~ms}$ to process and complete 1000 requests. And a single request only requires $0.561 \mathrm{~ms}$. Hence, this framework is capable of processing around 1784 requests in a single second. The rest of the frameworks were tested in the similar ways with different inputs and different number of requests. The response time and other details were noted down for the comparison in the form of tables. These tests were not only performed for the basic HTML websites but for various other coded websites including Php and Flask so that the framework does not fail in the realworld scenarios. Alongside the tables, the graphical representation for the same was available for the observational purpose. The Table 1 highlights the time taken by an HTML website to respond to the requests on all the various frameworks including the proposed one [11]. The number of users for which these tests were performed is five. 
Deploying a Framework to Improve the Performance of a Web Service

Table 1 Response Time of an HTML website on Different Frameworks

\begin{tabular}{lccc}
\hline \multicolumn{1}{c}{ Framework } & $\begin{array}{c}\text { Time per request } \\
(\mathbf{m s})\end{array}$ & $\begin{array}{c}\text { Time per request (across Requests per second } \\
\text { all concurrent request) } \\
\text { [(ms) }\end{array}$ & \\
\hline Proposed Framework & 11.210 & 0.561 & 1784.07 \\
LAMPP & 13.447 & 0.647 & 1235.14 \\
XAMPP & 13.447 & 0.647 & 1235.14 \\
Localhost & 15.453 & 1.453 & 688.42 \\
WAMP & 17.664 & 1.166 & 1010.37 \\
Cloud & 149.641 & 149.641 & 6.68 \\
\hline
\end{tabular}

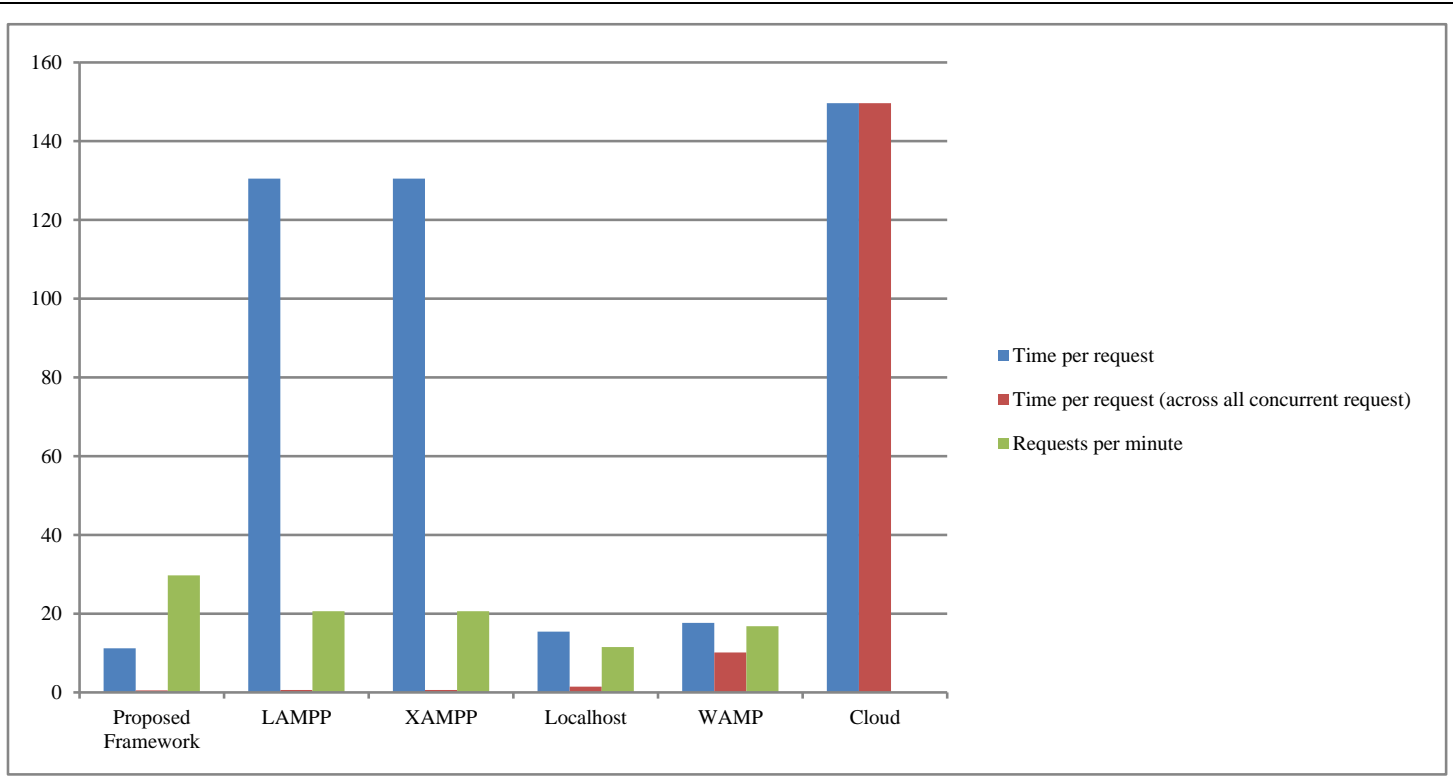

Figure 15. Response Time of an HTML website on Different Frameworks

Similarly, there is Table 2 depicting the response time of a PHP website on the following different frameworks with 5 users using it [12].

Table 2 Response Time of a PHP website on Different Frameworks

\begin{tabular}{lccc}
\hline Framework & $\begin{array}{c}\text { Time per } \\
\text { request } \\
(\mathbf{m s})\end{array}$ & $\begin{array}{c}\text { Time per request (across } \\
\text { all concurrent request) } \\
(\mathbf{m s})\end{array}$ & $\begin{array}{c}\text { Requests per second } \\
\text { [\#/sec] }\end{array}$ \\
\hline Proposed Framework & 1.790 & 0.358 & 2793.30 \\
LAMPP & 1.950 & 1.150 & 869.41 \\
XAMPP & 1.950 & 1.150 & 869.41 \\
Localhost & 1.984 & 1.984 & 1016.26 \\
WAMP & 2.450 & 0.445 & 4081.97 \\
Cloud & 152.658 & 152.658 & 6.55 \\
\hline
\end{tabular}




\section{S. Goyal and A. Gill}

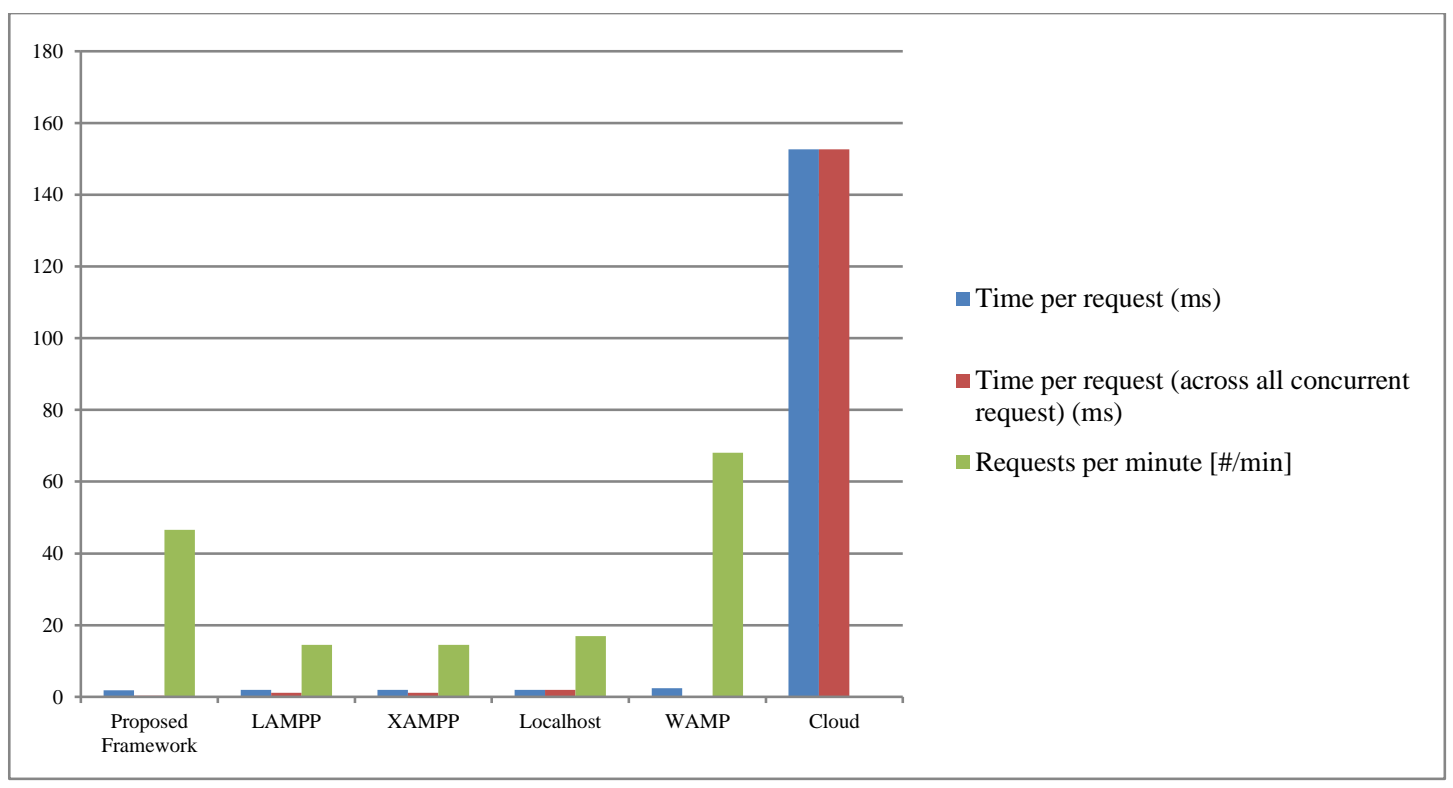

Figure 16. Response Time of a PHP website on Different Frameworks

The similar comparison was made between these frameworks on a Flask website with an equal number of users [13]. The Table 3 here displays the data for the same.

Table 3 Response Time of a FLASK website on Different Frameworks

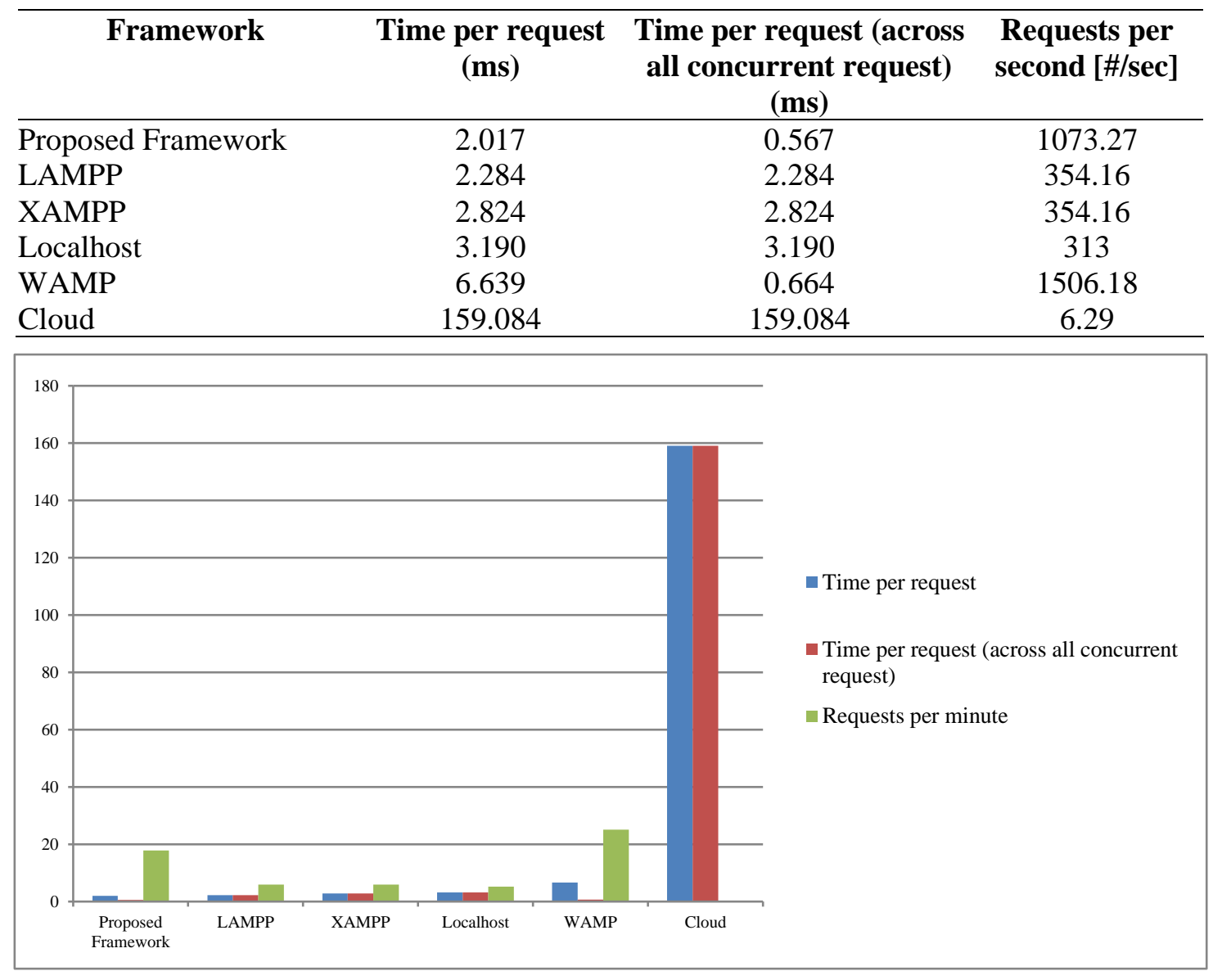

Figure 17. Response Time of a Flask website on Different Frameworks 
The observation of the data states that the Kube Devops Framework (the proposed frame work) provides the best performance. And, the results clear that amongst the existing frameworks, LAMPP secures the highest position. The same test had been performed for 2000 requests, 3000 and 4000 for all the frameworks. The pattern drawn from the results of these tests agrees with the first test.

\section{CONCLUSION}

The Kube Devops Framework (proposed framework) has been designed to improve the performance of the web services. The main feature that counts in measuring the performance is the response time of the web services. Hence, it has been the main objective of the paper to reduce the response time of these services. Since Kubernetes and Redis cache is open source, it helps in making the framework at low costs. The containerization with Kubernetes makes the framework portable. Kubernetes provides inbuilt load balancing and Redis cache provides the inbuilt code storage. These features in turn help the websites to respond in lesser time; hence this framework has successfully reduced the response time of the web services resulting in the improvement of the performance of the web services. The proposed framework is better than the existing ones based on parameters like cost, response time, and load balancing and added technologies. The new designed framework is more portable and platform independent. The results make it clear that the proposed framework can perform more efficiently even with large number of requests. There is Table 4 that depicts the various features in context of which the new proposed framework serves well than the existing ones. The following comparison helps in concluding that it's not only the technical figures and numbers that make the proposed framework better, but the ease and user friendliness are also the added factors which positively sums up in serving the purpose of the objective of this research paper. Since this framework offers lower response time, and lesser the response time, higher the speed of the website. Using this framework, the web service providers can attract more customers and users to their portal. Also, the customer friendly nature of the framework makes it easy to use for all types of customers, even if they're not very skilled. This framework serves its purpose fully and has attained the whole objective of the paper.

\section{REFERENCES}

[1] Ahmed, T. M., Bezemer, C., Chen, T., Hassan, A. E. and Shang, W. Studying the Effectiveness of Application Performance Management (APM) Tools for Detecting Performance Regressions for Web Applications: An Experience Report. IEEE/ACM 13th Working Conference on Mining Software Repositories, 2016, pp. 1-12.

[2] Alsmad, I. and Alda, S. Test Cases Reduction and Selection Optimization in Testing Web Services. Information Engineering and Electronic Business, 5, 2012, pp. 1-8.

[3] Arumugam. K. A. Software Testing Techniques \& New Trends. International Journal of Engineering Research \& Technology, 8(12), 2019, pp. 708-713.

[4] Khan. A. N. M, Mirza. M. A, Wagan. A. R, Shahid. M and Saleem. I. A Literature Review on Software Testing Techniques for Smartphone Applications. Engineering, Technology \& Applied Science Research, 10(6), 2020, pp. 6578-6583.

[5] Sathyavathy. V and Priyaa. S. D. Software Testing Techniques with Artificial Intelligence in Iot Applications", International Journal of Recent Technology and Engineering, 7(4), 2018, pp. 291-293.

[6] Bora, A. and Bezboruah T. A Comparative Investigation on Implementation of RESTful versus SOAP based Web Services. International Journal of Database Theory and Application, 8(3), 2015, pp. 297-312. 
[7] Jammalamadaka. K and Parveen. N. Holistic Research of Software Testing and Challenges", International Journal of Innovative Technology and Exploring Engineering, 8(6), 2019, pp. 1506-1521.

[8] Fageria, P. and Kaushik M. Research of Load Testing and Result Based on Load runner. International Journal of Civil Engineering, 1, 2014, pp. 1-4.

[9] Kelkar, D. and Kandalgaonkar, K. Analysis and Comparison of Performance Testing Tools. International Journal of Advanced Research in Computer Engineering \& Technology, 4(5), 2015, pp. 1880-1883.

[10] Kulkarni, V., Kotkar, P., Undale, M., Mankar, P., Mashal, K. and Komawar, K. Advanced Web Server Testing Tools. International Journal of Science and Research, 4(10), 2015, pp. $2064-$ 2066.

[11] Malik, M. F. and Khan, M. N. A. An Analysis of Performance Testing in Distributed Software Applications. International Journal Modern Education and Computer Science. 8(7), 2016, pp. 53-56.

[12] Mangal, D. and Mahajan, R. P. A Novel Approach for Performance Estimation of SOAP based Web Services. International Journal of Emerging Technology and Advanced Engineering. 2(2), 2012, pp. 81-86.

[13] Mohanty, R. K., Pattanayak, B. K. and Mohapatra, D. P. UML Based Web Service Regression Testing Using Test Cases: A Case Study: Asian Research Publishing Network. Journal of Engineering and Applied Sciences, 7(11), 2012, pp. 1416-1423. 